\title{
Numerical Modeling of Partially Coupled Problems of Thermoelasticity
}

\author{
Aziz A. Kalandarov ${ }^{1}$, Abdukayum Kalandarov ${ }^{2}$, Sayidulla Allayarov ${ }^{3}$, Sindorkul Kulmamatov ${ }^{4}$, \\ Sayid Islikov ${ }^{5}$, Abduraim Adilov ${ }^{6}$ \\ ${ }^{1}$ Head of the Department of Information Technology, Gulistan State University, Uzbekistan, abrorshox @mail.ru \\ ${ }^{2}$ Department of Mathematics, Gulistan State University, Uzbekistan, q_abduqayum@mail.ru \\ ${ }^{3}$ Dean of the Faculty of Physics and Mathematics, Gulistan State University, Uzbekistan, allayorov6464@mail.ru \\ ${ }^{4}$ Department of Information Technology, Gulistan State University, Uzbekistan, qulmamatov66@ inbox.ru \\ ${ }^{5}$ Department of Information Technology, Gulistan State University, Uzbekistan, dias6363@ mail.ru \\ ${ }^{6}$ Department of Information Technology, Gulistan State University, Uzbekistan, 60abdu19@ mail.du
}

\begin{abstract}
The paper proposes a new iterative approach for the numerical solution of partially coupled problems of thermo elasticity for isotropic bodies. The tasks are considered in two settings, dynamic and static. A discrete analogue of the boundary value problem is compiled on the basis of the finite difference method and an iterative process is performed, which allows you to find the values of the desired functions. It is assumed that, at the zero approximation, the values of the desired functions in the internal nodes are trivial. The essence of the proposed algorithm is demonstrated by numerically solving the one-dimensional dynamic and two-dimensional static thermo elasticity problems. The proposed algorithm can be applied for the numerical solution of thermoplastic coupled and unbound problems for isotropic and anisotropic bodies.
\end{abstract}

Key words: Computer simulation, deformation, stress, displacement, iterative process.

\section{INTRODUCTION}

Mathematical models describing the process of heat propagation were first considered in the works of Duhamel Neumann, in which it was assumed that the total deformation consists of elastic deformation and thermal expansion. When solving the problems of thermo elasticity, usually, the temperature distributions were determined previously based on the solution of the heat equation and, as it were, the equations of the theory of elasticity were solved with the temperature terms considered in combination with the volume forces. Related problems of thermo elasticity, within the framework of thermodynamic laws, were considered in the works of Biot M. [1], Youssef H.M. [2] and others. In [3] - [5], a numerical solution of coupled problems for isotropic and orthotropic bodies was considered. Further in [6], computer simulation of plastic stresses for transversely isotropic bodies was considered. The nonlinear properties of composite materials with radioisotope inclusions were studied in [7]. In
[8], electromagnetic voltages were investigated. A.A. Khaldjigitov and his students studied the issues of anisotropic plasticity taking into account temperature [9]-[10].

In many applied engineering and technical problems, the process of deformation of structures and their elements, taking into account temperature effects, can be described by model equations of two types, namely, in the form of a coupled or not coupled thermo mechanical problem. In an unrelated problem, the heat equation is solved separately, and its results are used as a well-known parameter in solving the basic model equations of thermo elasticity. Related problems, unlike unrelated problems, simultaneously solve the equations of thermo elasticity and the heat flux equation. Since deformations cause the appearance of temperature, and temperature causes the appearance of deformation, this approach allows a more adequate description of the process of thermo elasticity. In this paper, we consider partially related problems of thermo elasticity.

\section{FORMULATION OF THE PROBLEM}

A partially related problem of thermo elasticity can be considered in a dynamic or static setting.

\subsection{Dynamic problem}

Partially coupled dynamic thermo elasticity problem, consists of equations of motion

$$
\sum_{j=1}^{3} \frac{\partial \sigma_{i j}}{\partial x_{i}}+X_{i}=\rho \ddot{u}_{i}
$$

Duhamel-Neumann relations

$$
\sigma_{i j}=\lambda \theta \delta_{i j}+2 \mu \varepsilon_{i j}-(3 \lambda+2 \mu) \alpha\left(T-T_{0}\right) \delta_{i j}
$$

Cauchy relations

$$
\varepsilon_{i j}=\frac{1}{2}\left(u_{i, j}+u_{j, i}\right)
$$

and heat equations

$$
c_{\varepsilon} \dot{T}=\lambda_{0} T_{, i i}
$$

with initial

$$
\left.u_{i}\right|_{t=t_{0}}=\phi_{i},\left.\quad \dot{u}_{i}\right|_{t=t_{0}}=\psi_{i},\left.\quad T\right|_{t=0}=f
$$

and boundary conditions

$$
\left.u_{i}\right|_{\Sigma_{1}}=u_{i}^{0},\left.\quad \sum_{j=1}^{3} \sigma_{i j} n_{j}\right|_{\Sigma_{2}}=S_{i}^{o},\left.\quad T\right|_{\Sigma}=\varphi(t)
$$


where, $\sigma_{i j}$ - stress tensor, $\varepsilon_{i j}$ - strain tensor, $u_{i}$ - displacement components, $X_{i}$ - volume force, $\lambda, \mu$ - Lame constants, $T$-temperature, $T_{0}$-initial temperature, $\alpha$-corresponds to thermal expansion coefficient, $\theta=\varepsilon_{11}+\varepsilon_{22}+\varepsilon_{33}, \rho$-density of the body, $\delta_{i j}$ - delta Kronecker symbol, $n_{j}$ - external normal to the surface $\Sigma_{2}, S_{1}, S_{2}, S_{3}$ - components of the external load vector, $\lambda_{0}$-is the heat flow coefficient and $c_{\varepsilon}$-denotes heat at a constant deformation.

The boundary-value problem (1) - (6) in the one-dimensional case takes the form

$$
\begin{gathered}
\frac{\partial \sigma}{\partial x}+X=\rho \frac{\partial^{2} u}{\partial t^{2}} \\
\sigma=(\lambda+2 \mu) \varepsilon_{11}-(3 \lambda+2 \mu) \alpha\left(T-T_{0}\right) \\
\varepsilon_{11}=\frac{\partial u}{\partial x} \\
c_{\varepsilon} \frac{\partial T}{\partial t}=\lambda_{0} \frac{\partial^{2} T}{\partial x^{2}}
\end{gathered}
$$

with appropriate initial and boundary conditions

$$
\begin{gathered}
\left.u\right|_{t=t_{0}}=\phi,\left.\quad \frac{\partial u}{\partial t}\right|_{t=t_{0}}=\psi,\left.T\right|_{t=t_{0}}=f \\
\left.u\right|_{x=0}=u^{0},\left.u\right|_{x=l}=u^{\prime},\left.T\right|_{x=0}=\varphi_{1},\left.T\right|_{x=l}=\varphi_{1}
\end{gathered}
$$

Equations (7)-(10), after some transformations, can be written in the following form with respect to displacements and temperature

$$
\left.\begin{array}{c}
(\lambda+2 \mu) \frac{\partial^{2} u}{\partial x^{2}}-(3 \lambda+2 \mu) \alpha \frac{\partial T}{\partial x}=\rho \frac{\partial^{2} u}{\partial t^{2}} \\
c_{\varepsilon} \frac{\partial T}{\partial t}=\lambda_{0} \frac{\partial^{2} T}{\partial x^{2}}
\end{array}\right\}
$$

with initial and boundary conditions

$$
\left.\begin{array}{c}
\left.u\right|_{t=t_{0}}=\phi \quad,\left.\quad \frac{\partial u}{\partial t_{i}}\right|_{t=t_{0}}=\psi \quad,\left.\quad T\right|_{t=t_{0}}=f \\
\left.u\right|_{x=0}=u^{0},\left.u\right|_{x=l}=u^{\prime},\left.T\right|_{x=0}=\varphi_{1},\left.T\right|_{x=l}=\varphi_{1}
\end{array}\right\}
$$

In the system of differential equations (13), the second equation is independent of displacement, and can be solved separately independently of the first. For this reason, the system of equations (13-14) will be called a partially-connected boundary-value problem, as mentioned above.

\subsection{Static problem}

Neglecting the dynamic terms in the boundary value problem (1) - (6), i.e. inertial terms, we obtain

$$
\left.\begin{array}{c}
\sum_{j=1}^{3} \frac{\partial \sigma_{i j}}{\partial x_{i}}+X_{i}=0 \\
\sigma_{i j}=\lambda \theta \delta_{i j}+2 \mu \varepsilon_{i j}-(3 \lambda+2 \mu) \alpha\left(T-T_{0}\right) \delta_{i j} \\
\varepsilon_{i j}=\frac{1}{2}\left(u_{i, j}+u_{j, i}\right) \\
T_{i i}=0
\end{array}\right\}
$$

And boundary conditions

$$
\left.u_{i}\right|_{\Sigma_{1}}=u_{i}^{0},\left.\sum_{j=1}^{3} \sigma_{i j} n_{j}\right|_{\Sigma_{2}}=S_{i}^{0},\left.T\right|_{\Sigma}=T^{0} .
$$

For simplicity, we consider problem (15) - (17) in the two-dimensional case. After several transformations, equations (15) and (17) can be written in the following form, respectively

$$
\left.\begin{array}{c}
(\lambda+2 \mu) \frac{\partial^{2} u}{\partial x^{2}}+\mu \frac{\partial^{2} u}{\partial y^{2}}+(\lambda+\mu) \frac{\partial^{2} v}{\partial x \partial y}-(3 \lambda+2 \mu) \alpha \frac{\partial T}{\partial x}+X_{1}=0 \\
(\lambda+2 \mu) \frac{\partial^{2} v}{\partial y^{2}}+\mu \frac{\partial^{2} v}{\partial x^{2}}+(\lambda+\mu) \frac{\partial^{2} u}{\partial x \partial y}-(3 \lambda+2 \mu) \alpha \frac{\partial T}{\partial y}+X_{2}=0 \\
\frac{\partial^{2} T}{\partial x^{2}}+\frac{\partial^{2} T}{\partial y^{2}}=0
\end{array}\right\}
$$

with the following boundary conditions

$$
\left.u\right|_{\Gamma}=\bar{u} \quad,\left.\quad v\right|_{\Gamma}=\bar{v} \quad,\left.\quad T\right|_{\Gamma}=\phi(x, y) .
$$

\section{NUMERICAL METHOD}

\subsection{Numerical method for a dynamic problem}

Replacing the partial derivatives in equations (13) with finite differences, we can find that

$$
\begin{gathered}
(\lambda+2 \mu) \frac{u_{i+1, j}-2 u_{i, j}+u_{i-1, j}}{h_{1}^{2}}-(3 \lambda+2 \mu) \alpha \frac{T_{i+1, j}-T_{i-1, j}}{2 h_{1}}= \\
=\rho \frac{u_{i, j+1}-2 u_{i, j}+u_{i, j-1}}{\tau^{2}} \\
c_{\varepsilon} \frac{T_{i, j+1}-T_{i, j}}{\tau}=\lambda_{0} \frac{T_{i+1, j}-2 T_{i, j}+T_{i-1, j}}{h^{2}}
\end{gathered}
$$

solving difference equations (20)-(21) with respect to $u_{i, j+1}$ and $T_{i, j+1}$, we can find that

$$
\begin{gathered}
u_{i, j+1}=\frac{\tau^{2}}{\rho}\left((\lambda+2 \mu) \frac{u_{i+1, j}-2 u_{i, j}+u_{i-1, j}}{h_{1}^{2}}-(3 \lambda+2 \mu) \alpha \frac{T_{i+1, j}-T_{i-1, j}}{2 h_{1}}\right)+ \\
+2 u_{i, j}-u_{i, j-1} \\
T_{i, j+1}=\frac{\tau \lambda_{0}}{c_{\varepsilon}} \frac{T_{i+1, j}-2 T_{i, j}+T_{i-1, j}}{h^{2}}+T_{i, j} .
\end{gathered}
$$

The initial and boundary conditions with respect to the nodal points have the form

$$
\left.\begin{array}{c}
u_{i}^{0}=\phi_{i}, \quad \frac{u_{i}^{1}-u_{i}^{0}}{\tau}=\psi_{i}, \quad T_{i}^{0}=f_{i}, \quad i=\overline{0, N} \\
u_{0}^{j}=\bar{u}^{j}, u_{N}^{j}=\tilde{u}^{j}, T_{0}^{j}=\varphi_{1}^{j}, T_{N}^{j}=\varphi_{2}^{j}, \quad j=\overline{0, M}
\end{array}\right\}
$$

Using the nodal values of the functions $u_{i}^{0}, u_{i}^{1}$ and $T_{0}^{j}, T_{N}^{j}$ from the initial and boundary conditions, on the initial two layers, one can find the desired quantities using the recurrence relations (22) and (23).

Recall that the solution of problem (13) - (14) based on explicit finite-difference schemes was reduced to recurrence relations (22-23).

Now, let us consider how the iterative method can be applied to solve a partially coupled dynamic one-dimensional problem (13-14). To do this, replacing the partial derivatives in equations (13) with finite difference relations, we have

$$
\begin{gathered}
(\lambda+2 \mu) \frac{u_{i+1, j}-2 u_{i j}+u_{i-1, j}}{h^{2}}-(3 \lambda+2 \mu) \alpha \frac{T_{i+1, j}-T_{i-1, j}}{2 h}= \\
=\rho \frac{u_{i j}-2 u_{i, j-1}+u_{i, j-2}}{\tau^{2}} \\
c_{\varepsilon} \frac{T_{i, j}-T_{i, j-1}}{\tau}=\lambda_{0} \frac{T_{i+1, j}-2 T_{i, j}+T_{i-1, j}}{h^{2}} .
\end{gathered}
$$


Note that the right side of equation (25) differs from the right side of difference equation (20) and the left side of equation (26) differs from the left side of equation (21).

Solving the difference equations (25)-(26) with respect to $u_{i j}$ and $T_{i, j}$, respectively, we can find that

$$
\begin{gathered}
u_{i j}=\frac{(\lambda+2 \mu) \frac{u_{i+1, j}+u_{i-1, j}}{h^{2}}-\gamma \frac{T_{i+1, j}-T_{i-1, j}}{2 h}+\rho \frac{2 u_{i, j-1}-u_{i, j-2}}{\tau^{2}}}{\frac{2(\lambda+2 \mu)}{h^{2}}+\frac{\rho}{\tau^{2}}} \\
T_{i, j}=\frac{\lambda \tau}{c_{\varepsilon}} \frac{T_{i+1, j}-2 T_{i, j}+T_{i-1, j}}{h^{2}}+T_{i, j-1} \cdot
\end{gathered}
$$

Next, based on equations (27) and (28), we organize an iterative process

$$
\begin{gathered}
u_{i, j}^{(k+1)}=\frac{(\lambda+2 \mu) \frac{u_{i+1, j}^{(k)}+u_{i-1, j}^{(k)}}{h^{2}}-\gamma \frac{T_{i+1, j}-T_{i-1, j}}{2 h}+\rho \frac{2 u_{i, j-1}-u_{i, j-2}}{\tau^{2}}}{\frac{2(\lambda+2 \mu)}{h^{2}}+\frac{\rho}{\tau^{2}}} \\
T_{i, j}^{(k+1)}=\frac{\lambda_{0} \tau}{c_{\varepsilon}} \frac{T_{i+1, j}^{(k)}-2 T_{i, j}^{(k)}+T_{i-1, j}^{(k)}}{h^{2}}+T_{i, j-1}
\end{gathered}
$$

Using the initial and boundary conditions (24) on each layer in time $t$ for $j=2,3, \ldots$ from the iterative relations (29) - (30) one can find the values of the desired functions.

\subsection{Numerical method for a static problem}

Replacing the derivatives with finite-difference relations in equations (29), we can find the finite-difference equations

$$
\begin{aligned}
& (\lambda+2 \mu) \frac{u_{i+1, j}-2 u_{i, j}+u_{i-1, j}}{h_{1}^{2}}+(\lambda+\mu) \frac{v_{i+1, j+1}-v_{i-1, j+1}-v_{i+1, j-1}+v_{i-1, j-1}}{4 h_{1} h_{2}}+ \\
& \mu \frac{u_{i, j+1}-2 u_{i, j}+u_{i, j-1}}{h_{2}^{2}}-(3 \lambda+2 \mu) \alpha \frac{T_{i+1, j}-T_{i-1, j}}{2 h_{1}}=0 \\
& (\lambda+2 \mu) \frac{v_{i, j+1}-2 v_{i, j}+v_{i, j-1}}{h_{2}^{2}}+(\lambda+\mu) \frac{u_{i+1, j+1}-u_{i-1, j+1}-u_{i+1, j-1}+u_{i-1, j-1}}{4 h_{1} h_{2}}+ \\
& +\mu \frac{v_{i+1, j}-2 v_{i, j}+v_{i-1, j}}{h_{1}^{2}}-(3 \lambda+2 \mu) \alpha \frac{T_{i, j+1}-T_{i, j-1}}{2 h_{2}}=0 \\
& \frac{T_{i+1, j}-2 T_{i, j}+T_{i-1, j}}{h_{1}^{2}}+\frac{T_{i, j+1}-2 T_{i, j}+T_{i, j-1}}{h_{2}^{2}}=0 .
\end{aligned}
$$

We solve the system of difference equations (31) - (32) with respect to $u_{i, j}, v_{i, j}$ and $T_{i, j}$, respectively, and construct the following iterative process

$$
\begin{aligned}
u_{i, j}^{(k+1)}= & {\left[(\lambda+2 \mu) \frac{u_{i+1, j}^{(k)}+u_{i-1, j}^{(k)}}{h_{1}^{2}}+(\lambda+\mu) \frac{v_{i+1, j+1}^{(k)}-v_{i-1, j+1}^{(k)}-v_{i+1, j-1}^{(k)}+v_{i-1, j-1}^{(k)}}{4 h_{1} h_{2}}+\right.} \\
& \left.+\mu \frac{u_{i, j+1}^{(k)}+u_{i, j-1}^{(k)}}{h_{2}^{2}}-\alpha(3 \lambda+2 \mu) \frac{T_{i+1, j}-T_{i-1, j}}{2 h_{1}}\right] /\left[\frac{2(\lambda+2 \mu)}{h_{1}^{2}}+\frac{2 \mu}{h_{2}^{2}}\right] \\
v_{i, j}^{(k+1)}= & {\left[(\lambda+2 \mu) \frac{v_{i, j+1}^{(k)}+v_{i, j-1}^{(k)}}{h_{2}^{2}}+(\lambda+\mu) \frac{u_{i+1, j+1}^{(k)}-u_{i-1, j+1}^{(k)}-u_{i+1, j-1}^{(k)}+u_{i-1, j-1}^{(k)}}{4 h_{1} h_{2}}+\right.} \\
& \left.+\mu \frac{v_{i+1, j}^{(k)}+v_{i-1, j}^{(k)}}{h_{1}^{2}}-\alpha(3 \lambda+2 \mu) \frac{T_{i, j+1}-T_{i, j-1}}{2 h_{2}}\right] /\left[\frac{2(\lambda+2 \mu)}{h_{2}^{2}}+\frac{2 \mu}{h_{1}^{2}}\right]
\end{aligned}
$$

$$
T_{i, j}^{(k+1)}=\frac{\frac{T_{i+1, j}^{(k)}+T_{i-1, j}^{(k)}}{h_{1}^{2}}+\frac{T_{i, j+1}^{(k)}+T_{i, j-1}^{(k)}}{h_{2}^{2}}}{\frac{2}{h_{1}^{2}}+\frac{2}{h_{2}^{2}}} .
$$

In equations (33-35), the superscript $k$ is the number of (successive approximations) iterations. For $k=0$, the values of the functions $u_{i, j}^{(0)}, v_{i, j}^{(0)}$ and $T_{i, j}^{(0)}$ are known on the boundary $\Gamma$, according to boundary conditions (30), at the internal nodal points, they are trivial.

\section{TEST PROBLEMS}

\begin{tabular}{|c|c|c|c|c|c|}
\hline \multicolumn{6}{|c|}{ for $\varepsilon=0.001$} \\
\hline$t^{x}$ & 0 & 0.1 & 0.2 & 0.3 & 0.4 \\
\hline 0 & 0 & 4.63525 & 8.81678 & 12.13525 & 14.26585 \\
\hline 0.01 & 0 & 4.62958 & 8.80599 & 12.12041 & 14.24839 \\
\hline 0.02 & 0 & 4.62392 & 8.79523 & 12.10559 & 14.23098 \\
\hline 0.03 & 0 & 4.61827 & 8.78448 & 12.09079 & 14.21358 \\
\hline 0.04 & 0 & 4.61263 & 8.77374 & 12.07601 & 14.19621 \\
\hline 0.05 & 0 & 4.60699 & 8.76301 & 12.06125 & 14.17885 \\
\hline
\end{tabular}

\subsection{Dynamic problem}

As an example, we solve a dynamic partially connected problem under the following boundary and initial conditions

$$
\begin{aligned}
& \left.u\right|_{t=0}=0,\left.\quad \frac{\partial u}{\partial t}\right|_{t=0}=0,\left.\quad T\right|_{t=0}=T_{0} \sin \frac{\pi x_{i}}{l},\left.\quad u\right|_{x=0}=0,\left.\quad u\right|_{x=l}=0, \\
& \left.T\right|_{x=0}=0,\left.\quad T\right|_{x=l}=0
\end{aligned}
$$

and initial constants

$\lambda=0.8, \mu=0.5, \alpha=0.05, \rho=0.9, c_{\varepsilon}=3.2$,

$\lambda_{0}=0.04, T_{0}=15, l=1, N=10, h=0.1, \tau=0.01$.

Table 1: Values of the function $u(x, t)$ (iterative method) for $\varepsilon=0.001$

\begin{tabular}{|c|c|c|c|c|c|}
\hline \multicolumn{1}{|l|}{$\mathrm{x}$} & 0 & 0.1 & 0.2 & 0.3 & 0.4 \\
\hline 0 & 0 & 0 & 0 & 0 & 0 \\
\hline 0.01 & 0 & -0.00042 & -0.00035 & -0.00026 & -0.00014 \\
\hline 0.02 & 0 & -0.00163 & -0.00141 & -0.00102 & -0.00054 \\
\hline 0.03 & 0 & -0.00358 & -0.00316 & -0.00230 & -0.00121 \\
\hline 0.04 & 0 & -0.00623 & -0.00559 & -0.00407 & -0.00214 \\
\hline 0.05 & 0 & -0.00949 & -0.00869 & -0.00633 & -0.00333 \\
\hline
\end{tabular}

Table 2: Values of the function $u(x, t)$ (explicit scheme)

\begin{tabular}{|c|c|c|c|c|c|}
\hline \multicolumn{1}{|l|}{$\mathrm{x}$} & 0 & 0.1 & 0.2 & 0.3 & 0.4 \\
\hline 0 & 0 & 0 & 0 & 0 & 0 \\
\hline 0.01 & 0 & -0.00042 & -0.00035 & -0.00026 & -0.00014 \\
\hline 0.02 & 0 & -0.00165 & -0.00142 & -0.00103 & -0.00054 \\
\hline 0.03 & 0 & -0.00369 & -0.00318 & -0.00231 & -0.00121 \\
\hline 0.04 & 0 & -0.00646 & -0.00564 & -0.00410 & -0.00216 \\
\hline 0.05 & 0 & -0.00992 & -0.00880 & -0.00640 & -0.00336 \\
\hline
\end{tabular}

Table 3: Temperature values $T(x, t)$ (iterative method) 
Table 4: Temperature values $T(x, t)$ (explicit scheme)

\begin{tabular}{|c|c|c|c|c|c|}
\hline \multicolumn{1}{|r|}{$\mathrm{x}$} & 0 & 0.1 & 0.2 & 0.3 & 0.4 \\
\hline 0 & 0 & 4.63525 & 8.81678 & 12.13525 & 14.26585 \\
\hline 0.01 & 0 & 4.62958 & 8.80599 & 12.12041 & 14.24839 \\
\hline 0.02 & 0 & 4.62392 & 8.79522 & 12.10558 & 14.23096 \\
\hline 0.03 & 0 & 4.61826 & 8.78445 & 12.09076 & 14.21355 \\
\hline 0.04 & 0 & 4.61261 & 8.77371 & 12.07597 & 14.19615 \\
\hline 0.05 & 0 & 4.60697 & 8.76297 & 12.06119 & 14.17878 \\
\hline
\end{tabular}
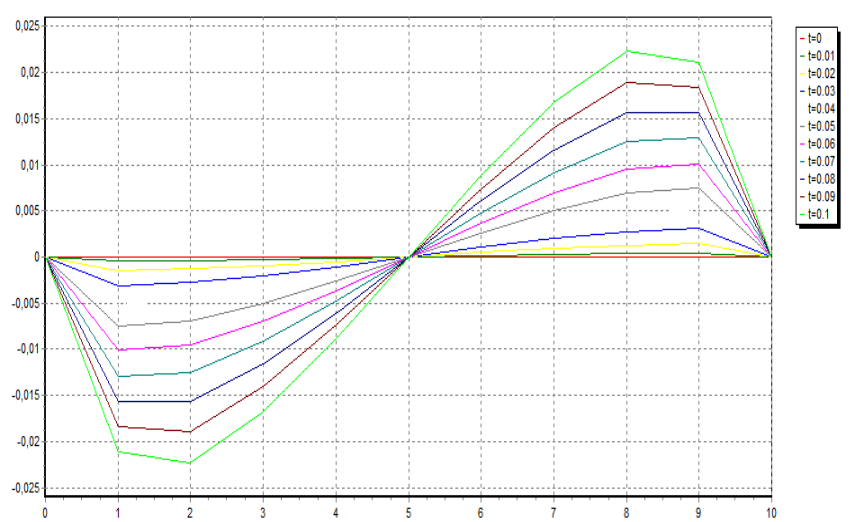

Figure 1: Graph of the distribution of the function $u(x, t)$ along the $O X$ axis (iterative method) for $\varepsilon=0.001$
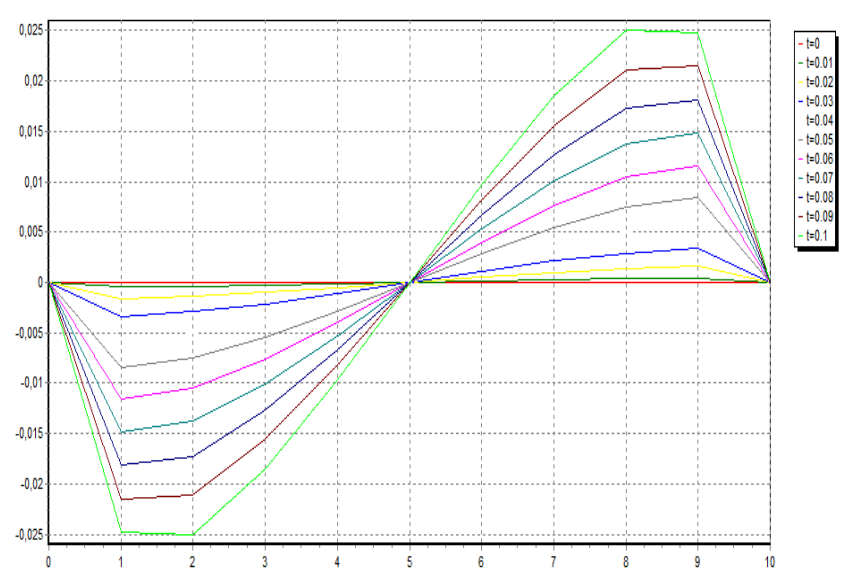

Figure 2: Graph of the distribution of the function $u(x, t)$ along the $O X$ axis (explicit scheme)

From the Tables 1-4 and Figures 1-2 it can be seen that the numerical values of the approximate solutions obtained by the two methods are quite close.

\subsection{Static problem}

Note that according to the boundary conditions on all sides of the rectangle, the displacement values are equal to zero; on the sides perpendicular to the $O Y$ axis, a sinusoidal temperature is specified. On the other two sides, it is assumed that the temperature is zero. The values of the desired functions, at internal points, at zero approximation are considered zero at $k=0$.

The problem was solved with the following parameter values $\lambda=0.7, \mu=0.3, \alpha=0.125, l_{1}=l_{2}=1, N 1=N 2=10$.

$$
\begin{aligned}
& \left.u_{i j}^{(0)}\right|_{\Gamma}=0,\left.\quad v_{i j}^{(0)}\right|_{\Gamma}=0, T_{0 j}^{(0)}=0, T_{N_{1} j}^{(0)}=0, \\
& T_{i 0}^{(0)}=T_{0} * \sin \frac{\pi x_{i}}{l_{1}}, \quad T_{i N_{2}}^{(0)}=T_{0} * \sin \frac{\pi x_{i}}{l_{1}}, \quad T_{0}=15 .
\end{aligned}
$$

Table 5: Values of the function $u(x, y)$ for $\varepsilon=0.0001$

\begin{tabular}{|c|c|c|c|c|c|}
\hline \multicolumn{1}{|c|}{$\mathrm{x}$} & 0 & 0.1 & 0.2 & 0.3 & 0.4 \\
\hline 0 & 0 & 0 & 0 & 0 & 0 \\
\hline 0.1 & 0 & -0.08237 & -0.10713 & -0.09261 & -0.05264 \\
\hline 0.2 & 0 & -0.10103 & -0.13932 & -0.12418 & -0.07167 \\
\hline 0.3 & 0 & -0.10343 & -0.14491 & -0.13088 & -0.07614 \\
\hline 0.4 & 0 & -0.10227 & -0.14353 & -0.13014 & -0.07593 \\
\hline 0.5 & 0 & -0.10156 & -0.14491 & -0.12924 & -0.07545 \\
\hline
\end{tabular}

Table 6: Values of the function $v(x, y)$ for $\varepsilon=0.0001$

\begin{tabular}{|c|c|c|c|c|c|}
\hline $\mathrm{x}$ & 0 & 0.1 & 0.2 & 0.3 & 0.4 \\
\hline 0 & 0 & 0 & 0 & 0 & 0 \\
\hline 0.1 & 0 & 0.01652 & 0.05859 & 0.09696 & 0 \\
\hline 0.2 & 0 & 0.02940 & 0.07584 & 0.11972 & 0 \\
\hline 0.3 & 0 & 0.02902 & 0.06551 & 0.09965 & 0 \\
\hline 0.4 & 0 & 0.01746 & 0.03719 & 0.05536 & 0 \\
\hline 0.5 & 0 & 0 & 0 & 0 & 0 \\
\hline
\end{tabular}

Table 7: Temperature $T(x, y)$ for $\varepsilon=0.0001$

\begin{tabular}{|c|c|c|c|c|c|}
\hline \multicolumn{1}{|c|}{$\mathrm{x}$} & 0 & 0.1 & 0.2 & 0.3 & 0.4 \\
\hline 0 & 0 & 4.63525 & 8.81678 & 12.13525 & 14.26585 \\
\hline 0.1 & 0 & 3.51870 & 6.69296 & 9.21207 & 10.82944 \\
\hline 0.2 & 0 & 2.74661 & 5.22436 & 7.19071 & 8.45319 \\
\hline 0.3 & 0 & 2.24344 & 4.26728 & 5.87341 & 6.90461 \\
\hline 0.4 & 0 & 1.95997 & 3.72809 & 5.13128 & 6.03218 \\
\hline 0.5 & 0 & 1.86847 & 3.55404 & 4.89171 & 5.75056 \\
\hline
\end{tabular}

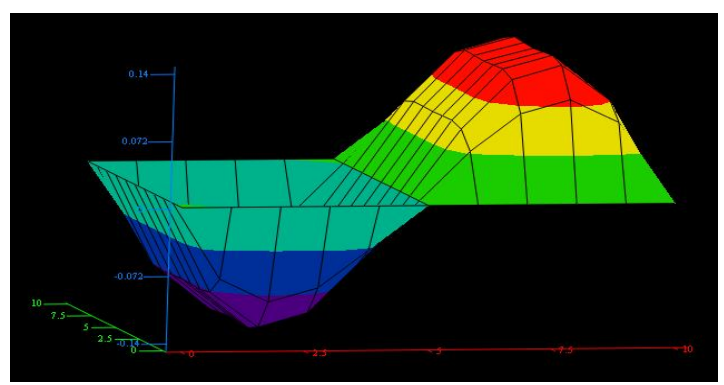

Figure 3: The distribution graph of the function $u(x, y)$ inside the rectangle for $\varepsilon=0.0001$

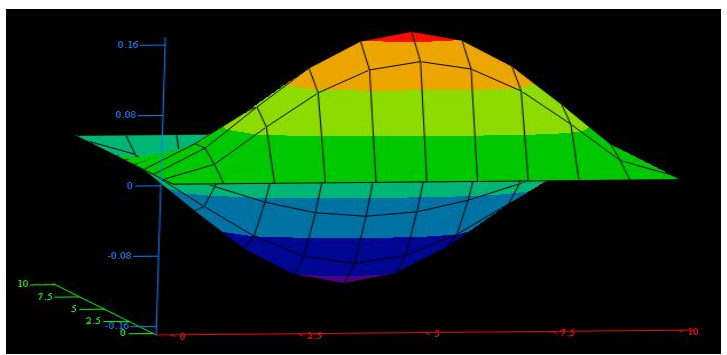

Figure 4: The distribution graph of the function $v(x, y)$ inside the rectangle for $\varepsilon=0.0001$ 


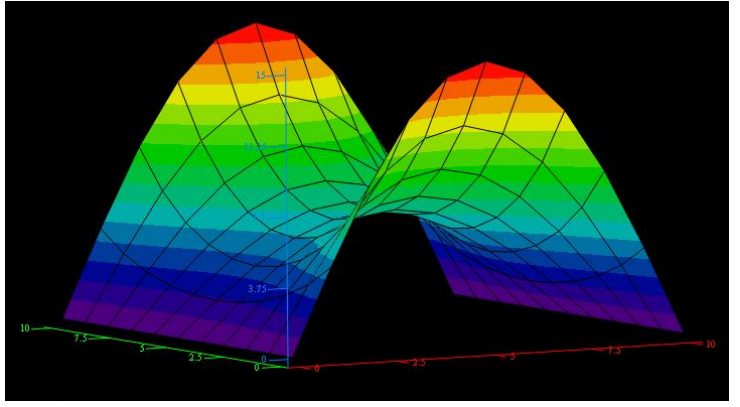

Figure 5: The distribution graph of the function $T(x, y)$ inside the rectangle for $\varepsilon=0.0001$

The displacement values $u(x, y)$ and $v(x, y)$ can be seen in Tables 5-6 and Figures 3-4, which are equal to zero at the edges of the rectangle, which is consistent with the given boundary conditions. The values of the function are symmetrical with respect to the midlines. The maximum temperature value $T=15$ is reached in the center of the side where the temperature of the sinusoidal shape is applied (Table 7). In Figures 3-5, we can analyze the distributions of the components of the displacements $u(x, y), v(x, y)$ and the temperature $T(x, y)$ in the rectangle.

\section{CONCLUSION}

The article presents a new approach for the numerical solution of partially coupled boundary problems of thermo-elasticity. These tasks are considered in dynamic and static setting. The dynamic problem was solved by two methods for comparing numerical results. The results obtained are very close, this ensures the reliability of the numerical results and the solution method. For a static problem, symmetric boundary conditions are considered. The numerical results obtained are also symmetrical. The proposed numerical solution technique can be applied to solve thermoplastic problems.

\section{REFERENCES}

1. M. Biot. Thermo elasticity and irreversible thermo-dynamics. J. Appl. Phys., 27, 240-253. 1956. https://doi.org/10.1063/1.1722351

2. H. M. Youssef and A.S. Al-Felali. Generalized thermo elasticity Problem of Material Subjected to Thermal Loading Due to Laser Pulse. Applied Mathematics, 2012, 3, pp. 142-146. https://doi.org/10.4236/am.2012.32022

3. A.A. Khaldjigitov, A.A. Qalandarov, NikM.A.Asri Long and Z. Eshquvatov. Numerical solution of $1 D$ and 2D thermoplastic coupled problems. International journal of modern physics, 2012, Vol. 9, pp. 503-510. https://doi.org/10.1142/S2010194512005594

4. A.A. Kalandarov and M.R. Babadjanov. Numerical simulation of the coupled dynamic thermoplastics problem for orthotropic bodies. International journal of computer science and Mobile computing, vol. 8 Issue 9, September 2019, pp. 182-189.

5. A.A. Qalandarov and A.A. Khaldjigitov. Mathematical and numerical modeling of the coupled dynamic thermo elastic problem for isotropic bodies. TWMS
Journal of Pure and Applied Mathematics. Vol. 11, N. 1, 2020, pp. 119-126.

6. A.M. Pulatov, A.M. Ikramov and A.A. Khaldjigitov. Computer Modeling of Elastoplastic Stress state of Fibrous Composites with hole//Coupled Systems Mechanics, An International Journal, Techno-Press Ltd., Yuseong-gu Daejeon, Korea, 2019, Vol.8, №4, pp. 299-313.

7. V. Savchenko, O. Vorobiov, O. Tkalenko, O. Polonevych, G. Shuklin, M. Trembovetskyi, V. Zaika and M. Konopliannykova. Influence of the composite materials nonlinear properties with radioisotope inclusions on reflected radiation. International Journal of Advanced Trends in Computer Science and Engineering, 2019, vol. 8, N.6, pp. 2716-2720. https://doi.org/10.30534/ijatcse/2019/05862019

8. M. Iasechko, V. Larin, S. Salkutsan, L. Mikhailova, O. Kozak and O. Ochkurenko. Formalized model descriptions of modified solid-state plasmalike materials to protect radio-electronic means from the effects of electromagnetic radiation. International Journal of Advanced Trends in Computer Science and Engineering, 2019, vol. 8, N. 3, pp.393-398. https://doi.org/10.30534/ijatcse/2019/09832019

9. A.A. Khaldjigitov, Nik M.A. Asri Long., A. Qalandarov and Z. Eshquvatov. Mathematical and numerical modelling of the thermoplastic coupled problem. International conference on mathematical sciences and statistics 2013. Singapore, Springer, 2014, pp. 69-75. https://doi.org/10.1007/978-981-4585-33-0_8

10. A.A. Khaldjigitov, Y.S. Yusupov, R.S. Khudazarov and D.A. Sadullaeva. On the thermoplasticity constitutive relations for isotropic and transversely isotropic materials. International Journal of Mechanical and Production Engineering Research and Development (IJMPERD), 2019, Vol.9, Issue 4, pp. 467-478. https://doi.org/10.24247/ijmperdaug201946 